\section{CPC-103 PHARMACIST-MANAGED INSULIN TITRATION VERSUS STANDARD CARE IN A VASCULAR SURGERY UNIT}

doi:10.1136/ejhpharm-2013-000276.560

'L Canadell Vilarrasa, ' $\mathrm{L}$ Castillo Palomares, ${ }^{2} \mathrm{~N}$ Argiles Mattes, ${ }^{2} \mathrm{EC}$ Rollan Saint Amand, ${ }^{2} \mathrm{JC}$ Sepulveda Grisales, ${ }^{2} \mathrm{R}$ Garcia Vidal, ${ }^{2} \mathrm{~L}$ Sanchez Parada, ${ }^{2} \mathrm{~J}$ Torrent Pou, ${ }^{1} \mathrm{M}$ Canela Subirada. 'Hospital Universitari Joan XXIII de Tarragona, Pharmacy, Tarragona, Spain; ${ }^{2}$ Hospital Universitari Joan XXIII de Tarragona, Vascular Surgery, Tarragona, Spain

Background Hyperglycemia is a prevalent situation in hospitalised patients and it has been associated with higher morbidity and mortality. Poor glycemic control is related to higher costs due to longer hospital stays and higher rates of complications. A large percentage of vascular surgery patients in our hospital have diabetes mellitus with a poor glycemic control.

Purpose To assess the impact of a collaborative, pharmacistmanaged insulin titration programme compared to standard medical care on glycemic control in patients with neuropathic diabetic foot ulcers in vascular surgery unit.

Materials and Methods It was established a new protocol to control glycemic levels in hyperglycemic patients in our hospital. To assess its effectiveness a prospective cohort study to compare pharmaceutical intervention of insulin titration to standard medical care was implanted. 30 patients were recorded and evaluated, 15 subjects were included as control (standard medical care before implantation of insulin protocol) and 15 in the pharmacist-managed group (insulin titration programme). Patients were selected consecutively on admission to the vascular surgery unit, the control group, one month prior to the implementation of the protocol and the rest one month later. In both groups it was registered: age, diabetes mellitus type, blood glucose levels, diet and drug treatment. Student t test was used to evaluate the glycemic values between groups.

Results Both groups were analysed and compared: $67 \%$ of subjects from control group were men vs $92 \%$ from the intervention group. No significant differences were found in the composition between both groups $(p>0.05)$ respect of age, diabetes mellitus type and diet. The pharmacist-managed group showed a lower glycemic level compared to standard medical care group $(123 \mathrm{mg} / \mathrm{dl}$ vs $170 \mathrm{mg} / \mathrm{dl}$ respectively; $p<0.044)$. The hyperglycemic levels were more frequent in control group than intervention group (78\% vs $35 \%$ ). No statistics differences were found with hypoglycemic situations ( $2 \%$ vs $4.5 \% \mathrm{p}=0.1$ )

Conclusions At the end of the study period, the intervention group patients had better glycemic control. Pharmacist-provider collaboration can result in significant clinical improvements when compared to standard care glycemic control in diabetic patient in a surgical unit.

No conflict of interest.

\section{CPC-104 PHARMACISTS AND CLINICAL TRIALS: PERSPECTIVES AND RESULTS AT THE MEDICAL ONCOLOGY OPERATIONS UNIT OF THE G. RUMMO HOSPITAL, BENEVENTO}

doi:10.1136/ejhpharm-2013-000276.561

'A Melillo, ${ }^{2} F$ Ruggiero, 'L Russo. 'Università degli Studi di Salerno, Scuola di Specializzazione Farmacia Ospedaliera, Fisciano (Sa), Italy; ${ }^{2}$ A.O. "Guido Salvini"-- Rho (MI), Farmacia, Corso Europa 25020017 RHO (MI), Italy

Background The rules on Controlled Clinical Trials require the expertise of a pharmacist specialising in internal monitoring of ongoing trials in the Operations Unit.

Purpose To highlight the role of the pharmacist dedicated to research projects, who sees that trials are conducted in accordance with GCP and in compliance with applicable regulations.

Materials and Methods From January 2010 the pharmacy has created a database to monitor all studies approved by the Ethics
Committee, both observational and experimental. Having a dedicated pharmacist has led to: proper storage of drugs, completing the application form accompanying the samples, storage of electronic and paper documentation of the experimental samples, fitting directly in Pharmacy, randomization of patients enrolled and completing the Drug Accountability.

Results 40 clinical trials have been conducted, 26 of which were conducted in the Oncology OU, 3 in Pulmonary and 8 in Cardiology, 1 in Rheumatology, 2 in Dermatology. As regards the preparation of the antiblastic treatments, the treatment setting provided by the experimental protocols accounted for $5 \%$ of all cancer preparations performed in the pharmacy. $83 \%$ of the studies (33 studies) were for profit, non-profit research accounted for only $17 \%$ of the studies. In 2012 the number of for-profit studies increased compared to 2010; we hope these will be particularly useful to point out any problems of current clinical practise.

Conclusions The dedicated pharmacist can ensure that research is conducted properly, both the management of experimental drugs and collaboration with the clinical evaluations related to routes of administration, any incompatibilities, monitoring of side effects and/or adverse events, interactions with associated therapies. In conclusion it is evident that the multidisciplinary approach and sharing of expertise with the medical and nursing staff encourages adherence to protocols.

No conflict of interest.

\section{CPC-105 PHARMACOECONOMIC ASPECTS OF THE TREATMENT OF RHEUMATOID ARTHRITIS WITH TUMOUR NECROSIS FACTOR ALPHA ANTAGONISTS: A SOCIETAL PERSPECTIVE}

doi:10.1136/ejhpharm-2013-000276.562

${ }^{1} \mathrm{C}$ Marongiu, ${ }^{2} \mathrm{C}$ Francisco, ${ }^{3} \mathrm{~V}$ Modena, ${ }^{4} \mathrm{~V}$ Azzolini, ${ }^{5 P} \mathrm{P}$ Milla, ${ }^{5} \mathrm{~L}$ Cattel. ${ }^{1 / v r e a}$ Hospital and School of Hospital Pharmacy - University of Turin, Pharmacy, Ivrea, Italy; ${ }^{2}$ Ivrea Hospital and School of Hospital Pharmacy - University of Turin, Pharmacy, Ivrea, Italy; ${ }^{3}$ University Hospital A.O. Città della Salute e della Scienza, Rheumatology, Turin, Italy; ${ }^{4} / v r e a$ Hospital, Rheumatology, Ivrea, Italy; ${ }^{5}$ School of Hospital Pharmacy - University of Turin, Scienza e Tecnologia del Farmaco Dep., Turin, Italy

Background Rheumatoid arthritis (RA) is an autoimmune disorder, affecting $1 \%$ of the population, characterised by pain, joint swelling and progressive destruction of joint tissue. EULAR (European League Against Rheumatism) recommends the use of Tumour Necrosis Factor alpha antagonists (anti-TNF $\alpha$ ) if methotrexate or Disease Modifying Antirheumatic Drugs fail. Anti-TNF $\alpha$ treatment imposes a significant financial burden on hospital budgets.

Purpose To perform a pharmacoeconomic investigation in the Piedmont region (Italy) to identify the cost of the illness RA. To analyse the payer's and societal perspectives, investigating direct costs associated with health care use and indirect costs related to productivity loss.

Materials and Methods A multidisciplinary group, rheumatologists, hospital pharmacists and pharmacoeconomists, was established to perform a pharmacoeconomic evaluation of the direct and indirect costs of RA, by a systematic literature review. Afterward, we plan a perspective, observational, multicentre, cost-effectiveness analysis of RA biological drugs, involving 100 patients. Each patient will be recorded, every three months for one year, through personal data, disease duration and characterization, systemic manifestations and comorbidities, prescribed biological medicines. A questionnaire will be submitted, in order to assess direct and indirect costs.

Results 40 existing pharmacoeconomic evaluations were critically appraised: the overall mean costs of RA amounted to about $€ 15,000$ per year, while the direct annual costs of RA were on average about $€ 4,000$. The greatest burden of RA costs was the indirect costs. From a societal perspective the superior clinical outcomes achieved 
with anti-TNF $\alpha$ are worth their higher costs. The most favourable incremental cost-effectiveness ratio was for etanercept compared to methotrexate

Conclusions The cost-effectiveness of an intervention depends on the maximum the decision makers are willing to pay for an extra unit of health effect. It should be considered that treatments with anti-TNF $\alpha$, in a societal perspective, decrease the use of health resources and increase productivity.

No conflict of interest.

\section{CPC-106 PHARMACOKINETIC DRUG-DRUG INTERACTIONS DUE TO TREATMENT WITH AMIODARONE - A PRACTICAL APPROACH}

doi:10.1136/ejhpharm-2013-000276.563

N Carstens. Apotekene Vest, Sjukehusapoteket i Haugesund, Haugesund, Norway

Background The drug amiodarone has a complex pharmacokinetic profile and can be a challenge to use due to the high potential for drug-drug interactions.

Purpose To identify and submit proposals for handling drug-drug interactions for patients treated with amiodarone. In addition we would like to highlight the fact that drug interactions can occur even if amiodarone is administered as only a single IV dose, and the effect on further treatment. The purpose was also to prepare proposals for management and follow ups of interactions in the clinic.

Materials and Methods Before the ward round the pharmacist carried out medicines reviews for the 25 patients who were included. They were all treated with amiodarone at admission or during hospitalisation. Input was given on the clinically significant interactions identified. For patients treated with warfarin in addition to IV amiodarone the INR values were observed through the entire hospital stay for any signs of a drug-drug interaction.

Results The pharmacist had 54 inputs referring to interactions with amiodarone, of which 41 were taken into account. The inputs led to dose reductions, changes of drugs and monitoring of blood values. Case reports showed that interactions do occur after IV amiodarone treatment and these lead to uncertain and variable drug efficacy over time.

Conclusions Based on results from the study and a literature search, general advice for handling interactions due to amiodarone and further treatment were prepared. The recommendations were endorsed by the consultant Cardiologist.

\section{Abstract CPC-106 Table 1}

Advice for avoiding Drug-Related Problems DRPs due to treatment with amiodarone Warfarin Reduce/give half-dose warfarin at start-up. Monitor the INR values (1)

Digitoxin Give half dose digitoxin/digoxin and monitor digitoxin/digoxin determined by procedure (2)

Simvastatin No doses above $20 \mathrm{mg}$ or switch to another statin. (3)

Atorvastatin Note the dose! No clear recommendations, but maximum $40 \mathrm{mg}$

Metoprolol Bradycardia? The dose may be adjusted (4)

\section{General advice}

When admitted from other hospitals

Note in the drug curve if recently treated with amiodarone!

\section{Discharge summaries}

Explain why the GP should follow up the blood values; INR, digitoxin/digoxin and possibly CK.

1. Edvin SB et al, An evaluation of early pharmacodynamic response after simultaneous initiation of warfarin and amiodarone.

2. Laer $\mathrm{S}$ et al, Digitoxin intoxication during concomitant use of amiodarone.

3. Marot A et al, Concomitant use of simvastatin and amiodarone resulting in severe rhabdomyolysis: a case report and literature review

4. Fukumoto et al, Effect of amiodarone on the serum concentration/dose ratio of metoprolol in patients with cardiac arrhythmias

No conflict of interest.

\section{CPC-107 PHARMACOTHERAPY FOLLOW-UP IN CHRONIC HEPATITIS C PATIENTS TREATED WITH BOCEPREVIR OR TELAPREVIR}

doi:10.1136/ejhpharm-2013-000276.564

I Cañamares-Orbis, C García-Muñoz, M Campo-Angora, JM Ferrari-Piquero, I EscribanoValenciano. Hospital Universitario 12 de Octubre, Hospital Pharmacy, Madrid, Spain

Background The approval for the clinical use of direct-acting antivirals in 2011 (boceprevir [BOC] and telaprevir [TLV], viral NS3 protease inhibitors) has increased recovery rates by up to $70 \%$. However follow-up of these patients is necessary due to adverse effects (AEs) and the high cost of the treatment.

Purpose To follow up the pharmacotherapy in chronic hepatitis C virus genotype-1.

(VHC-1) patients treated with triple therapy (TT): BOC or TLV, ribavirin and peg-interferon.

To evaluate the efficacy of the treatment and describe the pharmacological handling of severe AEs.

Materials and Methods Prospective study (from 01/01 to 30/9/2012) was carried out in the Pharmacy Department. VHC-1 patients who started TT were included. All of them had at least one viral load (VL) determination (BOC at week 8 and TLV at week 4).

A hospital pharmacist interviewed the patient at the first day treatment and provided oral and written information about how to take the drugs and their potential AEs.

Later, we analysed the compliance of the treatment to the guidelines of Spanish Agency for Drugs. Patient data (age, sex, basal LV at week 4 and week 8 , previous treatment response, fibrosis and haemoglobin levels) were collected from electronic clinical histories and outpatient software.

Results 35 patients were included (22 TLV and 13 BOC), 28 had initial VL $>800000 \mathrm{IU} / \mathrm{mL}$. 34 patients had fibrosis grade $\geq 3.13$ patients were treatment-naive, 22 had been treated previously ( 9 non-responders, 8 relapsers, 5 partial responders). 2 BOC patients obtained fast viral response vs. 4 TLV patients, and 7 BOC patients had undetectable VL at the week 8 cheque-up vs. 16 TLV patients at week 4 cheque-up.

5 patients (4 with BOC) discontinued treatment, one due to severe toxicity and 4 due to lack of efficacy. TT was effective and adhered to the guidelines in $84 \%$ patients.

The most frequent AEs were asthenia, anaemia and dermatological reactions (mainly with TLV). 9 patients presented grade 3 anaemia and were treated with erythropoiesis-stimulating agents (EEAs) (31\% BOC vs. 23\% TLV).

Conclusions The safety profiles of BOC and TLV found in our study were similar to those published in clinical trials. Despite not being a comparative study, we observed that more people in the TLV group reached undetectable VL after 4 or 8 weeks (91\% TLV vs. $69 \%$ BOC). Patients treated with BOC had earlier suspended the TT because of lower effectiveness and higher occurrence of grade 3 anaemia that required EAAs.

No conflict of interest.

\section{CPC-108 PHARMACY INTERVENTIONS UNDERTAKEN IN AN INTENSIVE CARE UNIT SPECIALISING IN WOMEN'S HEALTH}

doi:10.1136/ejhpharm-2013-000276.565

${ }^{1}$ P Mazzola, ${ }^{1}$ LS Costa, ${ }^{1}$ AT Rodrigues, ${ }^{2}$ AER Silva, ${ }^{2}$ ACA Bernardes, ${ }^{2} N M O$ Silva, ${ }^{3}$ AFO Neto. ${ }^{1}$ Faculty of Medical Sciences, Department of Clinical Pathology, Campinas, Brazil; ${ }^{2}$ Women's Hospital Prof. Dr. Jose Aristodemo Pinotti - CAISM/UNICAMP, Pharmacy, Campinas, Brazil; ' ${ }^{3}$ Women's Hospital Prof. Dr. Jose Aristodemo Pinotti - CAISM/ UNICAMP, Intensive Care Unit, Campinas, Brazil

Background Pharmaceutical interventions can prevent drugrelated problems and possible prescription errors. They thus 